\title{
DOI: https://doi.org/10.24297/jap.v19i.9048
}

\section{Revolutionary Approach for Fusion of the Classic, Relativity and Quantum field Theories: Sayed's Acceleration Equation and Probable Violation of $E=m^{2}$}

\author{
Sayed A. El-Mongy \\ Ex. V.Chairman of Egypt Nuclear and Radiological Regulatory Authority (ENRRA). \\ sayedelmongy@hotmail.com
}

\begin{abstract}
Expansion of the universe is a divine fact in the glorious Quran. The accelerated expansion of the universe is one of the physics mysteries and challenges. This article is a try to find an answer to this ambiguity. A simple fusion and merging of the Newton, Einstein and quantum field equations were carried out to clarify this topic. Innovative equations correlating the acceleration $\left(A_{s}\right)$, cosmological constant $(\Lambda)$, vacuum energy density $(\rho)$ and distance (d) was deduced. It can be concluded that Sayed's acceleration constant $\left(A_{s}\right)$ is proportional to $(N / \rho)$, $\left(1 / 8 m c^{2}\right)$ and $\left(1 / \pi d^{2}\right)$. The derivative equation reveals a probable violation of the mass-energy formula $\left(E=m c^{2}\right)$; the speed of light might be $12.5 \%$ more. This disparity may be due to antimatter contribution; neutrinoantineutrino, $\beta^{-} \beta^{+}$annihilation and/or a predicted unrecognized very light particle in the atom nucleus. The Sayed's acceleration constant $\left(A_{s}\right)$ and $\left(A_{s} / \Lambda\right)$ ratio were calculated and found to be $6.33825 \times 10^{-8} \mathrm{~m} / \mathrm{s}^{2}$ and $5.7620475 \times 10^{+44} \mathrm{~m}^{3} / \mathrm{s}^{2}$ respectively. Using Sayed's equations, the calculated acceleration in planck scale is matched with the declared $5.56081 \times 10^{51} \mathrm{~m} / \mathrm{s}^{2}$ value. The The calculated recession velocity at $1 \mathrm{Mpc}$ was found to be $6.5192677 \times 10^{8} \mathrm{~m} / \mathrm{s}$.and the cosmological constant $(\Lambda)$ is as measured; $\sim 1.1 \times 10^{-52} \mathrm{~m}^{-2}$
\end{abstract}

Keywords: Sayed's acceleration $\left(A_{s}\right)$ constant/ Unified equations/ Probable violation of $E=m c^{2}$.

\section{Introduction.}

The accelerated expansion of the universe is one of the most puzzling and tantalizing mysteries to the modern physics and human thinking. In the year 2011, the Noble prize for physics was gratned to observers of this ambiguity issue; discovery of the accelerating expansion of the Universe(1). Development of the universe since the the Big Bang is shown in fig. 1 (2).

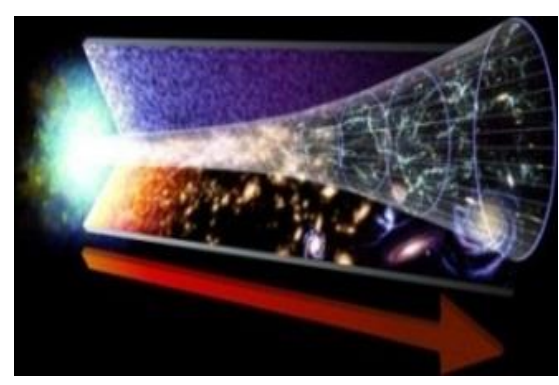

Fig. (1): History of the universe from the Big Bang till now

The cosmologists consider that the energy density made up of 4\% baryonic matter (observed), $23 \%$ dark matter (invisible), and $73 \%$ cosmological constant (dark energy) (3). If dark energy gets stronger with time, space will eventually tear itself apart, resulting in a Big Rip scenario, tearing atoms themselves apart. Alternatively, if dark energy increases in magnitude but reverses its sign; from positive to negative, the universe will be recollapsed and end in a Big Crunch (4).

This article is an ambitious try to find explanation to the accelerated expansion of the universe through fusion and correlation of the Newton, Relativity and quantum field theories together. 


\section{Review of the some cosmological observations and publications.}

- In this paragraph, some observations and recent publications will be tanged as follows:

- The Big Blunder of Einstein, as he said, was in 1917 by introducing the cosmological constant $(\Lambda)$ in the field equations to predict static (non-dynamic) universe (5).

- Hubble discovered expansion of the universe in 1929. While, accelerated expansion of the universe was observed by two teams of scientists in 1998 (6).

- Hubble's constant has debatable values. The scientists calculated expansion rate of $67.4-74.0$ $\mathrm{km} / \mathrm{sec} / \mathrm{Mpc}(7,8)$. The universe might be $2 \mathrm{~B}$ yrs younger than the accepted age $(9)$.

- Cygnuis X-1 Black hole (S. Hawking loss of betting) was found to be bigger and faster than the old observations $(10,11)$. A magnetic field around a BH has observed (12).

- The infinitesimal gravitational force between two tiny gold spheres, a sesame seed size, separated by a few millimeters was recently measured (13).

- Neutrinos may have interaction with matter, the Antarctic ice sheet might have seen the first evidence of a rare neutrino-interaction process called the Glashow resonance (14).

- An article indicates, energy-mass equivalence is a little incomplete. It is more accurate to say that energy can convert into equal amounts of matter and antimatter (15).

- An article shows that Quasar light curves do not show the effects of time dilation (16).

- New theorem for arc length calculation; (ет) term in astrophysics was published(17).

\section{The equations fused, merged and correlated in this work.}

\section{III.a. Newton classic equations.}

When an external force acts on a body, it produces an acceleration of the body in the direction of the force. This is mathematically expressed as (18);

$F=m a$

(1) Where $F$ is the net force, $m$ is mass and $a$ is the acceleration $(a=F / m)$. The acceleration is also defined by change of velocity over time; $\mathrm{dv} / \mathrm{dt}$.

$F=G m_{1} m_{2} / d^{2}$

(2) Where, $F$ is the force of gravity between two bodies of masses $m_{1}$ and $m_{2}, G$ is the universal gavitational constant and $d$ is the distance between centers of the two masses. The $G$ constant can also be given as;

$G=F d^{2} / m_{1} m_{2}$

\section{III.b. The special and general relativity equations.}

The great physicist Einstein was not the first to equate forms of mass to energy, nor did he definitively prove the relationship $(19,20)$. The very famous equation $\left(E=m c^{2}\right)$ of Einstein special relativity was developed through different stages as can be seen from Table $1(19,20)$.

Table 1: Development of Mass-Energy Equivalence formula

\begin{tabular}{|l|l|l|l|}
\hline Scientist & Date & \multicolumn{2}{|l|}{ Formula } \\
\hline Oliver Heaviside & 1889 & $m=(4 / 3) E / c^{2}$ & $\mathrm{E}=(3 / 4) \mathrm{mc}^{2}$ \\
\hline Henri Poincaré & 1900 & $m_{e m}=E_{e m} / \mathrm{c}^{2}$ & $\mathrm{E}_{\mathrm{em}}=\mathrm{m}_{\mathrm{em}} \mathrm{c}^{2}$ \\
\hline F.Hasenöhrl & 1904 & $\mathrm{~m}_{\circ}=(8 / 3) \mathrm{E}_{\mathrm{em}} / \mathrm{c}^{2}$ & $\mathrm{E}_{\mathrm{em}}=(3 / 8) \mathrm{m}_{\circ} \mathrm{c}^{2}$ \\
\hline F.Hasenöhrl & early 1905 & $\mathrm{~m}_{\circ}=(4 / 3) \mathrm{E}_{\mathrm{em}} / \mathrm{c}^{2}$ & $\mathrm{E}_{\mathrm{em}}=(3 / 4) \mathrm{m}_{\circ} \mathrm{c}^{2}$ \\
\hline A.Einestein & 1905 & $\mathrm{~m}=\mathrm{E} / \mathrm{c}^{2}$ & $\mathrm{E}=\mathrm{m} \mathrm{c}^{2}$ \\
\hline Sayed El-Mongy & 2021 (this work) & $\mathrm{M}=8 \mathrm{E} / \mathrm{c}^{2}$ & $\mathrm{E}=1 / 8 \mathrm{mc}^{2}$ \\
\hline
\end{tabular}

The general relativity was declared in 1915 without the cosmological constant. The cosmological constant $\Lambda$ was introduced by Albert Einstein to keep static universe concept into general relativity in 1917. After discovery the 
expansion of the universe, He said that this was his Big Blunder. The Einestein field equation (EFE) is simply given by the following formula $(18,21)$ :

$$
R_{\mu v}-1 / 2 R g_{\mu v}+\Lambda G_{\mu v}=\left(8 \pi G / c^{4}\right) T_{\mu v}
$$

The Einstein equations relate on the one hand geometry, in the form of the Ricci tensor $R_{\mu v}$, the metric tensor $g_{\mu v}$ and the curvature $R$, and matter represented by the energy-momentum tensor $T_{\mu v}$. $G$ is Newton's gravitation constant and $\mathrm{c}$ is the speed of light. The derivation of Newtonian law of gravity can be achieved from general relativity as given in this reference (22).

\section{III.c. The Quantum field equation (QFE).}

According to quantum field theory (QFT), empty space is defined by the vacuum state which is a collection of quantum fields. All these quantum fields exhibit fluctuations in their ground state (lowest energy density) arising from the zero-point energy present everywhere in space. These zero-point fluctuations should act as a contribution to the cosmological constant $\Lambda$, but when calculations are performed these fluctuations give rise to an enormous vacuum energy. the energy density of vacuume can be expressed as follow $(18,21,23,24)$.

$\rho=\left(8 \pi G / c^{2}\right) \wedge$

(5) Where, $\rho$ is the energy density of vacuum $\left(\mathrm{Kg} / \mathrm{m}^{3}\right), c$ is the speed of light $(\mathrm{m} / \mathrm{s}), \Lambda$ is the Einestein cosmological constant $\left(\mathrm{m}^{-2}\right)$ and $G$ is the universal Newton's gravitational constant $\left(\mathrm{Nm}^{2} / \mathrm{kg}^{2}\right)$. The positive vacuum energy density resulting from a cosmological constant implies a negative pressure, and vice versa. If the energy density is positive, the associated negative pressure will drive an accelerated expansion of the universe, as observed. The discrepancy between theorized vacuum energy from quantum field theory and observed vacuum energy exceeding observation by $\sim 120$ orders of magnitude, "the worst theoretical prediction in the history of physics" and. is called the cosmological constant problem (CCP) $(2,6,18,21)$.

\section{Theoretical derivation of Sayed 's acceleration constant $\left(A_{s}\right)$ and equations.}

Nobody knows how to reconcile Quantum field theory and General relativity theories yet. That's what scientists working on "quantum gravity" are trying to do. Based on the classic Newtonion, Eniestein field equations and quantum field theory, the following simple fusion derivation was performed. The cosmological constant can be interpreted as the energy density of the vacuum. Specifically, if we introduce $(18,21,23,24)$ :

$T_{\mu v}^{\text {(vacuum) }}=\left(c^{4} \Lambda / 8 \pi G\right) g_{\mu v}$

By substitution taking into account energy momentum tensor of a perfect fluid, it would finally conclude that the energy density of the vacuum is:

$c^{2} \rho_{\text {vac }}=c^{4} \wedge / 8 \pi G$

This equation can be rewritten as;

$\rho_{\text {vac }}=c^{2} \wedge / 8 \pi G$

By substituting value of $G$ of equation 3 in equation 8 , considering $m_{1}=m_{2}$, one gets;

$\rho_{\text {vac }}=m^{2} c^{2} \Lambda / 8 \pi F d^{2}$

If we take into consideration the equation no. 1 to replace the value of $\mathrm{F}$ in equation 9 , it gets;

$\rho_{\text {vac }}=m^{2} c^{2} \Lambda / 8 \pi m a d^{2}$

This equation no. 10 can be rewritten as follows;

$\rho_{\text {vac }}=m c^{2} \wedge / 8 \pi a d^{2}$

This formula can also be rearranged in the next other different forms number 12,13 and 14 as;

$\rho_{\text {vac }}=\left(1 / 8 m c^{2}\right) \cdot(\Lambda / a) \cdot\left(1 / \pi d^{2}\right)$

$a / \Lambda=\left(1 / 8 m c^{2}\right) \cdot\left(1 / \rho_{\text {vac }}\right) \cdot\left(1 / \pi d^{2}\right)$

$\mathrm{a}=\left(\Lambda / \rho_{\text {vac }}\right) \cdot\left(1 / 8 m c^{2}\right) \cdot\left(1 / \pi d^{2}\right)$ 
The final form of this formula can be expressed in the following equation to be;

$A_{s}=(\Lambda / \rho) \cdot\left(1 / 8 m c^{2}\right) \cdot\left(1 / \pi d^{2}\right)$

Where, $\boldsymbol{A}_{\boldsymbol{s}}$ is called Sayed's acceleration constant, representing the acceleration rate $\left(\mathrm{m} / \mathrm{s}^{2}\right)$.

Considerning the kinetic energy defination for $\mathrm{v} \approx \mathrm{c}$, the formula can also be written as;

$A_{s}=(\Lambda / \rho) \cdot\left(1 / 2 m v^{2}\right) \cdot\left(1 / 4 \pi d^{2}\right)$

The speed of light can also be calculated basd on the formula 15 as;

$C=\sqrt{8 A_{s}} \pi d^{2} \rho / m \Lambda$

The cosmological constant $(\Lambda)$ can also be calculated as follows;

$\Lambda=\pi d^{2} \mathrm{~A}_{s} \rho\left(8 / m c^{2}\right)$

The ratio of the Sayed's acceleration $\left(\mathrm{A}_{s}\right)$ to the cosmological constant can be found by using;

$\mathrm{A}_{s} / \Lambda=(1 / \rho)\left(1 / 8 m c^{2}\right)\left(1 / \pi d^{2}\right)$

\section{Mathematical estimation of the Sayed's acceleration $A_{s}$ and other parameters}

Based on the derived equations of this work, some calculations were carried out using the following published values and constants $(18,21,23,24,25,26)$;

$C \approx 3 \times 10^{8} \mathrm{~m} / \mathrm{s} \quad$ (speed of light)

$M_{p}=2.176 \times 10^{-8} \mathrm{~kg} \quad$ (Planck mass).

$L_{p}=1.616 \times 10^{-35} \mathrm{~m} \quad$ (Planck length).

$\mathrm{T}_{\mathrm{p}}=5.39 \times 10^{-44} \mathrm{~s} \quad$ (Planck time).

$\rho=5.69 \times 10^{-27} \mathrm{~kg} / \mathrm{m}^{3}$ (Energy density of vacuum).

$\Lambda=1.1 \times 10^{-52} \mathrm{~m}^{-2} \quad$ (Observed value of the cosmological constant).

$1 \mathrm{Mpc}=3.0856776 \times 10^{22} \mathrm{~m} \quad($ Parsec; $1 \mathrm{pc}=3.26$ light year $)$.

Compton wavelength (proton) $=1.321 \times 10^{-15} \mathrm{~m} \quad$ (numerical value) .

Hydrogen atom (proton) mass $=1.6735 \times 10^{-27} \mathrm{~kg}$.

By substitution in our equations $15,17,18$ and 19 , the values of $\left(A_{s}\right),\left(A_{s} / \Lambda\right),(\Lambda),(c)$ and $(a \Lambda)$ were also calculated and presented in Table 2.

Table 2: The calculated $A_{s}$ and the cosmological values are based on hydrogen (proton) data

\begin{tabular}{|l|l|l|l|}
\hline Item & Reference value & Calculated by Sayed equations & $\%$ diff. \\
\hline Acceleration $\left(A_{s}\right)$, eq.15 & ----- & $6.33825231 \times 10^{-8} \mathrm{~m} / \mathrm{s}^{2}$ & New \\
\hline$\left(\mathrm{A}_{\mathrm{s}} / \Lambda\right)$, by eq.19 & ----- & $5.7620475 \times 10^{+44} \mathrm{~m}^{3} / \mathrm{s}^{2}$ & New \\
\hline $\begin{array}{l}\mathrm{V}=\mathrm{A}_{\mathrm{s}} . \mathrm{t} \text {, eq.1 } \\
\text { *Velocity of Recession }\end{array}$ & ----- & $6.5192677 \times 10^{8} \mathrm{~m} / \mathrm{s}$ & New \\
\hline The value $\left(\Lambda \mathrm{A}_{\mathrm{s}}\right)$ & ----- & & New \\
\hline Value of $(\Lambda)$; eq.18 & $1.1 \times 10^{-52} \mathrm{~m}^{-2}$ & $1.1 \times 10^{-52} \mathrm{~m}^{-2}$ & ---- \\
\hline **Light Speed; eq.17 & $\sim 3 \times 10^{8} \mathrm{~m} / \mathrm{s}$ & $2.93125943 \times 10^{8} \mathrm{~m} / \mathrm{s}$ & $2.34 \%$ \\
\hline
\end{tabular}

*The $t$ was calculated for speed of proton in vacuum; 1/100 light speed, at one Mpc.

$* * \mid t$ should be mentioned that the calculated (c) is considered as $E=1 / 8 \mathrm{mc}^{2}$, of $12.5 \%$. 
The hot and cold matter discrepancies are detectable only at accelerations $<10^{-8} \mathrm{~cm} / \mathrm{s}^{2}$ since they are all baryons (26). The following figure number 2 shows the correlation between the calculated recession velocities at different distances in $\mathrm{Mpc}\left(1 \mathrm{Mpc} \sim 3.08 \times 10^{22} \mathrm{~m}\right)$.

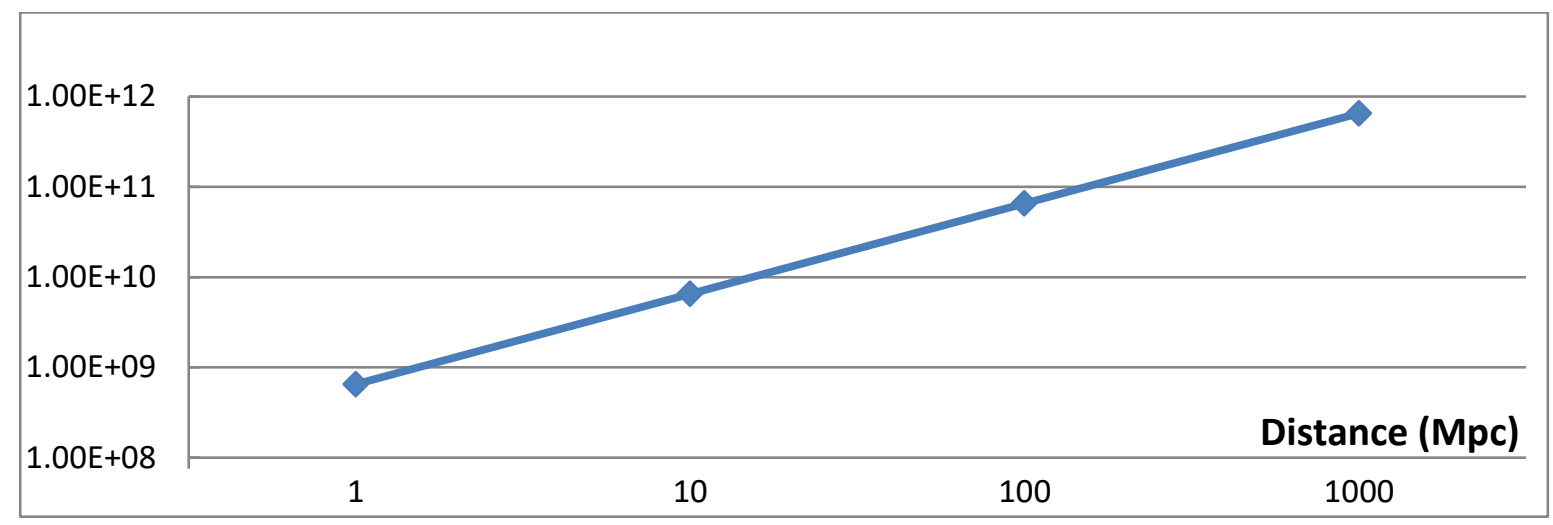

Fig. (2): The Distance and log of Recession Velocity $(\mathrm{m} / \mathrm{s})$ Correlation

Sayed's acceleration $\left(A_{s}\right)$ was also calculated, eqs. 1,15, using planck scale data as in Tables 3.

Table 3: The calculated acceleration based on planck length, mass and time values

\begin{tabular}{|l|l|l|l|}
\hline Item & *Reference value & Calculated by Sayed`s equation & $\%$ diff. \\
\hline Acceleration $\left(A_{s}\right)$ & $5.560815 \times 10^{51} \mathrm{~m} / \mathrm{s}^{2}$ & $5.7684564 \times 10^{+51} \mathrm{~m} / \mathrm{s}^{2}$ & $3.73 \%$ \\
\hline Recession velocity & $V=A_{s} \times T_{p}$ & $3.10895 \times 10^{8} \mathrm{~m} / \mathrm{s}$ & --- \\
\hline$\Lambda$ by eq. 18 & $1.1 \times 10^{-52} \mathrm{~m}^{-2}$ & $1.1030415 \times 10^{-52} \mathrm{~m}^{-2}$ & $0.276 \%$ \\
\hline
\end{tabular}

* The planck acceleration is the acceleration of an object which accelerates from zero to the speed of light during one planck time. The Compton wavelength $\left(\lambda_{c}=h / m c\right)$ is for a particle of mass $(m)$. This length is independent of the velocity of the particle. One $T_{p}$ is the time it would take a photon travelling at the speed of light to cross a distance equal to one Planck length.

\section{Results and discussion.}

Based on the main derived equation number 15, the following major crucial points might be stated and concluded to elucidate the mystery of the accelerated expansion of the universe:

$A_{s}$ is a new universal constant and is correlated in a direct function with $(\Lambda)$ and $(\rho)$.

$A_{s}$ is proportional to $\left(1 / 8 \mathrm{mc}^{2} ; \mathrm{m}=8 \mathrm{E} / \mathrm{c}^{2}\right)$; this term may indicates that the speed of light should be faster than the universal value by $(1 / 8=12.5 \%)$.

The probable explanation of the correctness and concordance of $E=m c^{2}$ calculations with many experimental observations (e.g. radioactive decay, nuclear fission and fusion,.....) might be attributed to the presence of simultanous contribution from other unconsidered sources such as antimatter interaction; neutrino-antineutrino (úv) and $\beta^{-} \beta^{+}$annihilation ( $2 \gamma$ photons) and a new predicted light particle in the nucleus. To keep the energymass matched with the correct observations. The $E=m c^{2}$ equation is suggested to be rewritten as given in the following equation ;

$E=m c^{2}\left(\Delta C_{\beta-\beta+}+\Delta C_{u ́ v}+\Delta C_{p p}\right)$

Where, the $\left(\Delta \mathrm{E}_{\beta-\beta+}\right),\left(\Delta \mathrm{E}_{\mathrm{uvv}}\right)$ and $\left(\Delta \mathrm{E}_{\mathrm{pp}}\right)$ representing the contribution of the value; $12.5 \%$. A new light particle $\left(\Delta \mathrm{E}_{\mathrm{pp}}\right)$ in the nucleus smaller than the quark might be play a role in this disparity too. In the light of our concluded remark, the following topics may consildate the issue;

-The scientists measured the bending of light due to gravity with shift angle of 1.75" (arc sec.) (18), but they did not simultanously measured its speed; the $12.5 \%$ shift might be observed. 
-A recent article recommends that it is more accurate to say that energy can convert into equal amounts of matter and antimatter (15).

-An article indicates that they measured muon neutrino (travelled $730 \mathrm{~km}$ From CERN to Italy) of velocity slightly exceed the speed of light (27); later, Prof A. Ereditato has resigned.

-My letter to the chief editor of nuclear physics journal entitle; Is it true that ${ }^{210} \mathrm{Po}$ is fissioned by antineutrinos ? (ISFP) process, was accepted and published in 2006 (28).

-Violation of matter-antimatter symmetry was observed in the 1960s, quarks and antiquarks don't behave in exactly the same way. Recently, the researchers report that they found evidence that neutrinos and antineutrinos oscillate in different ways $(29,30)$.

-A conference paper proves that the speed of light c decreases with cosmological time, and that c is proportional to the Hubble parameter $\mathrm{H}$ (31).

-The Scharnhorst effect (faster-than-c photon propagation in the Casimir vacuum) suggests that light particles may be able to travel faster than the accepted speed of light $(32,33)$.

-Muons and electrons might not experience the same fundamental interactions, contrary to Standard Model predictions; a new particle or force may be probable and suggested (34).

-The discovery of the diffuse Galactic positron annihilation radiation at $511 \mathrm{keV}$ from the inner Galaxy has shown that the positrons from the decay of radioactive nuclei produced by explosive nucleosynthesis in supernovae (SNe) are the largest and most likely source (35).

\section{Conclusion}

This new approach article reveals that Sayed's acceleration constant $\left(A_{s}\right)$ represents multiple correlated parameters to describe the universe accelerated expansion. The calculated $\left(A_{s}\right)$ value is $6.3382523 \times 10^{-8} \mathrm{~m} / \mathrm{s}^{2}$ $\left(\sim 6 \times 10^{-11} \mathrm{~km} / \mathrm{s}^{2}\right)$. The derived equation number 15 shows that the light speed should be more than the accepted well known value by (12.5\%). Predication of a new light particle in the nucleus and/or antimatter interactions; antineutrino and $\beta-\beta+$ annihilation, might be an exit to interpret violation of the mass-energy equivalence as the derived formula highlights. The calculated recession velocity using Sayed's acceleration equation is 3.10895 $\times 10^{8} \mathrm{~m} / \mathrm{s}$ in planck scale and $6.5192677 \times 10^{8} \mathrm{~m} / \mathrm{s}$ for hydrogen atom (proton) at $1 \mathrm{Mpc}$.

\section{Acknowledgment}

My Lord and creator, please accept my deep cordial thanks and ultimate mental respect. I tried in this article to clarify argument of the universe accelerated expansion.

\section{References}

1. https://www.nobelprize.org/prizes/physics/2011/press-release/, 2011.

2. Sabine Hossenfelder, "Is There Really A Cosmological Constant? Or Is Dark Energy Changing With Time?", Forbes, May 30, 2017. https://www.forbes.com/sites/startswithabang/2017/05/30/.

3. Anirudh Pradhan 1, Archana Dixit 2, Vinod Kumar Bhardwaj, "Barrow HDE model for Statefinder diagnostic in FLRW Universe", arXiv:2101.00176v1 [gr-qc] 1 Jan 2021.

4. Victoria Woollaston, "A Big Freeze, Rip or Crunch: how will the Universe end?", wired, https://www.wired.co.uk/article/how-will-universe-end. 2016.

5. Cormac O'Raifeartaigh \& Simon Mitton, "Interrogating the Legend of Einstein's "Biggest Blunder", Physics in Perspective volume 20, p 318-341, 2018.

6. Cosmological constant. www.wikipedia.com, last edited on 4 February 2021.

7. https://www.nasa.gov/feature/goddard/2019/new-hubble-constant-measurement-adds-to-mysteryof-universe-s-expansion-rate. 2019. 
8. Richard Panek, "How a Dispute over a Single Number Became a Cosmological Crisis ", Scientific American, https://www.scientificamerican.com/. March 2020.

9. Seth Borenstein, "Study finds the universe might be 2 billion years younger", Physic.org, 2019. https://phys.org/news/2019-09-universe-billion-years-younger.html. 2019.

10. Maria Temming, "The first black hole ever discovered is more massive than previously thought", Science News, Feb. 18, 2021.

11. Sayed A. El-Mongy," Hawking Radiation is Nothing: Developed Correlation of Entropy with Black Hole Area", Advances in physics, Vol.18, 2020. DOI: https://doi.org/10.24297/jap.v18i.8905.

12. Stephanie Pappas, "Magnetic Field around a Black Hole Mapped for the First Time", Scientific American, LiveScience on March 24, 2021. https://www.scientificamerican.com/article/magnetic-field-around-ablack-hole mapped-for-the-first-time/. 2021.

13. Ben Brubaker, "Physicists Measure the Gravitational Force between the Smallest Masses Yet", Scientific American, March 10, 2021.

14. Madeleine O'Keefe, "IceCube detection of high-energy particle proves 60-year-old physics theory", Wisconsin News, March 10, 2021. https://news.wisc.edu/

15. Don Lincoln, "The Perplexing Question of Missing Cosmic Antimatter", Forbes, 2021. https://www.forbes.com/sites/drdonlincoln/2021/02/10/the-perplexing-question-of-missing-cosmicantimatter/

16. O. I. Chashchina and Z. K. Silagadze, "Expanding space, quasars and St. Augustine's fireworks", arXiv:1409.1708v1 [gr-qc] 5 Sep 2014.

17. Sayed Ali El-Mongy," New Theorem and Formula for Circle Arc Length Calculations with Trigonometric Approach Application in Astrophysics:, Advances in physics, Vol.17, 2020. DOI: https://doi.org/10.24297/jap.v18i.8914.

18. TA-PEI CHENG, "Relativity, Gravitation, and Cosmology- A basic introduction", Oxford University Press, 2005.

19. Tony Rothman, "Was Einstein the First to Invent $E=m c^{2}$ ? ", Scientific American, August 24, 2015.

20. James Carter, "The Living Universe - How Einstein was Wrong about $\mathrm{E}=\mathrm{mc}^{2 "}$, The Living Universe, 2021. https://living-universe.com/how-einstein-was-wrong/.

21. The Cosmological Constant Problem, Physics 171, 2014.

22. Victor T. Toth, "How can we derive Newton's law of gravitation from Einstein's theory of relativity? "Quora, https://www.quora.com/. December 14. 2016.

23. Fran de Aquino, "The Observed Magnitude of Cosmological Constant directly derived from the Quantization Theory of Gravity", HAL Id: hal-02332672, 2019.

24. Christian Henke, "Quantum vacuum energy in general relativity", Eur. Phys. J. C, 78, 2018.

25. Moshe Carmeli and Tanya Kuzmenko," Value of the Cosmological Constant: Theory versus Experiment", https://cds.cern.ch/record/485959/files/0102033.pdf. 2001.

26. David E. Rosenberg "A Cyclical Universe". Yeshiva of Virginia, Richmond VA 23226 c 2016, December 7, 2016.

27. OPERA Collaboration, T. Adam et al., "Measurement of the neutrino velocity with the OPERA detector in the CNGS beam", arXiv: 1109.4897v4 [hep-ex], revised 12 Jul. 2012.

28. Sayed A. El-Mongy," Is it true that ${ }^{210} \mathrm{Po}$ is fissioned by antineutrinos", Letter to the Editor, Journal of Nuclear and Radiation Physics, Vol.1, No.1,. P77, 2006. 
29. Mike Wall, "Weird Neutrino Behavior Could Explain Long-standing Antimatter Mystery", Scientific American, April 16, 2020.

30. The T2K Collaboration, "Constraint on the Matter-Antimatter Symmetry-Violating Phase in Neutrino Oscillations", arXiv:1910.03887v3 [hep-ex], 25 Jan 2021.

31. Antonio Alfonso-Faus, "The Speed of Light and the Hubble Parameter: The Mass-Boom Effect", arXiv:0710.3039v3 [physics.gen-ph], Nov. 2008.

32. Klaus Scharnhorst, "Can light travel faster than light?", Philip Campbell, Editor of Physics World, BBC Science Magazine. http://www.nat.vu.nl/ scharnh/m16bbc.htm. 1990.

33. Stefano Liberati, Sebastiano Sonego, and Matt Visser,"Faster-than-c signals, special relativity, and causality", arXiv:gr-qc/0107091v2 14 Feb. 2002.

34. Daniel Garisto,"Unexplained Results Intrigue Physicists at World's Largest Particle Collider", Scientific American, on March 25, 2021. https://www.scientificamerican.com/

35. J. C. Higdon, R. E. Lingenfelter, and R. E. Rothschild, "The Galactic Positron Annihilation Radiation and the Propagation of Positron in the Interstellar Medium", The Astrophysical Journal, 698:350-379, June 10, doi:10.1088/0004-637X/698/1/350. 2009.

\section{Conflict of interests}

It must be mentioned that there is no any conflict of interest with anyone regarding this article.

\section{Biography}

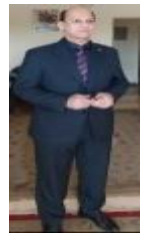

Professor Sayed was V. Chairman of Egypt Nuclear Regulatory Authority (ENRRA). He was Ph.D. fellow in Germany-Mainz, studying INAA and delayed neutrons assay using TRIGA-II reactor. He is currently a nuclear affairs consultant. He participated in meetings and conferences in; USA, Russia, Canada, EU States, S. Korea, the IAEA and Arab States. He has new theorems for black hole anatomy and arc length calculations with application in astrophysics and nuclear fields. 